\title{
Welcome to JMM Case Reports
}

Welcome to the first online issue of JMM Case Reports (http://jmmcr. sgmjournals.org/) - a newly launched gold open access online-only journal from the Society for General Microbiology (SGM; http://www.sgm. ac.uk/).

In early 2013, JMM Case Reports' sister journal - Journal of Medical Microbiology (JMM; http://jmm. sgmjournals.org/) - made the executive decision to stop accepting case reports for submission in the journal. The Editorial Board of JMM understood the value of case reports and their interest to readers; however, they felt that a journal dedicated solely to case reports would be more beneficial for both authors and readers alike. With this new journal in mind, JMM stopped accepting case reports for submission in September 2013 and the JMM Case Reports submission system opened shortly afterwards.

Case reports provide an educational tool for junior researchers and clinicians, whilst also keeping readers up to date on current research topics across the globe. Clinical case reports offer an immediate purpose to patient care, as well as a platform through which to highlight emerging infectious diseases and the new advances and challenges associated with these.

JMM Case Reports plans to publish original scientific case reports within the field of medical microbiology covering all types of micro-organism. Papers can be written from a broad perspective of microbiology (e.g. infectious disease, virology, mycology, bacteriology and parasitology), antimicrobials or microbial diagnostics, and should provide a focus on current research or serve an educational value.

It is expected that case reports will describe interesting and/or novel diagnoses, pathogenesis, investigations, treatment and/or management of infectious diseases in humans or animals. Papers should not merely describe observations made or focus solely on novelty of occurrence, but rather should include new insights, explanations of mechanisms, interpretations or other aspects conveying intellectual or academic originality.

In addition to case reports, SGM's new journal also welcomes submission of case series and case reviews, and more interactive paper types including case quizzes and entries for the Image of the Month competition. The journal strongly promotes the submission of clinical photographs, scans and other multimedia to support any cases that are submitted.

The first accepted paper in JMM Case Reports by Yamazaki et al. (2014) reports a case of hypocomplementaemic urticarial vasculitis in a child due to coxsackievirus type A9.

The new journal will publish four online issues a year and will also support fast publication times through a continuous publishing model, ensuring that articles are uploaded to the website as soon as the final version is ready, whilst allowing readers to access new content continuously.

JMM Case Reports has exceeded its proposed submissions target in 2013, and we welcome future submissions that meet the scope of the journal. Please do not hesitate to contact the Editorial Office if you have any queries (jmmcr@sgm.ac.uk).

SGM is waiving all Article Processing Charges in the journal's launch years; for further information regarding SGM's first open access online-only dedicated journal, please visit http:// jmmcr.sgmjournals.org/.

This has been an exciting time for SGM, as JMM Case Reports represents the first new journal launch in almost 50 years for the Society, and we look forward to the development of this exciting new and interactive journal.

Pete Borriello and Johannes Kusters, Co-Editors-in-Chief

Yamazaki, M., Sugai, K., Kobayashi, Y., Murashita, Y., Murashita, K., Saito, N., Hitoshi, N., Imagawa, T., Tshukagoshi, H. \& Kimura, H. (2014). A case of

hypocomplementaemic urticarial vasculitis in a child due to coxsackievirus type A9. JMM Case Rep 1, doi: 10.1099/jmmcr.0.000596-0.

DOI 10.1099/jmmcr.0.000919-0 http://jmmcr.sgmjournals.org 